\title{
Effects of a Horticultural Activity Program Based on Validation Therapy on the Mental Functions of Elderly Patients in Nursing Homes
}

\author{
Sook Lee* \\ Department of Horticultural Therapy and College of Nursing, Dankook University, Cheonan 31116, Korea
}

\section{ABSTRACT}

This study was conducted to investigate changes in the mental functions of the elderly in nursing facilities affected by a horticultural therapy program based on validation therapy. To meet the purpose, we conducted a horticultural therapy program based on validation therapy with 58 elderly participants (average age 79.12 26.84 , men and women) once a week, 50 minutes per session, in a total of 10 sessions. Participants were divided into the control and experimental group with convenience sampling. The program was carried out from March 15 to November 22, 2018. Vibraimage 8 pro(ELSYS, 2014) is a recent, psychologically based, emotional-recognition visual imaging technology that measures pixels microvibration in terms of digital frequency and amplitude parameters. To examine the effects of the horticultural therapy program based on validation therapy, Vibraimage was used to assess aggression, stress, tension, suspect, balance, charm, energy, self-regulation, inhibition, neuroticism and positive, negative, physiological domains of mental functions before and after program. As a result, the mean score of the positive domain in the control group significantly decreased from 63.89 \pm 5.09 to 60.74 \pm 5.48 , but it decreased without statistical significance in the experimental group from $63.98 \pm 5.45$ to $61.39 \pm 6.02$. The mean score of neuroticism in the experimental group significantly decreased from 31.64 10.94 to $22.87 \pm 13.79$. Moreover, the mean score of the physiological domain in the experimental group also significantly decreased from $25.08 \pm 6.27$ to $19.42 \pm 8.80$. Accordingly, horticultural therapy program based on validation therapy can be utilized as a program to promote mental health, especially maintaining positive mental health function of the eldery, helping those who live in long-term care facilities enjoy a happier life more happier.

Keywords: complementary medicine, elderly facility, emotion, horticultural therapy, Vibraimage

\section{Introduction}

The elderly population in Korea is rapidly increasing, with senior citizens aged 65 and above taking up 14.3\% of the entire population in 2018, and the percentage is expected to exceed $41.0 \%$ by 2060 (Statistics Korea, 2011). The elderly entering nursing homes, which are elderly care facilities, show decreased physical functions such as chronic illnesses and dementia and thus need continuous care service such as healthcare service, which is why the number of long-term elderly care facilities is increasing (Ministry of Health and Welfare, 2019). Moreover, since elderly people in nursing homes are isolated from the outside, they lack accessibility to family and face difficulty in going out, thereby resulting in psychological problems such as sense of isolation and depression, as well as deteriorated quality of life (Lee, 2014). In particular, the remarkable change in mental function along with geriatric, chronic diseases lead to deterioration of intellectual capacity and sensory functions. Thus, chronic diseases, physical functions and

\footnotetext{
This study is funded by the research fund for professors at Dankook University in 2017.

Received: November 14, 2019, Revised: November 19, 2019, Accepted: December 3, 2019

First author: Sook Lee, moonlight@dankook.ac.kr, (1) https://orcid.org/0000-0002-8451-8573

*Corresponding author: Sook Lee, moonlight@dankook.ac.kr, (D) https://orcid.org/0000-0002-8451-8573
} 
mental health of the elderly in long-term care nursing homes are becoming worse, and the limited space and various rules in the facility are especially having more effect on the elderly's mental health (Song, 2012).

Validation therapy promotes human dignity, mental security and self-esteem by fully accepting words and behaviors of the elderly with dementia under the assumption that their words and behaviors have significant meaning to them even though they may seem confusing to others. This is a non-pharmacological intervention that encourages patients to express their emotions by accepting their view rather than maintaining the sense of reality. Previous studies that applied validation therapy mostly examined its effects on depression, quality of life, problem behavior symptoms and cognitive functions of the elderly with dementia living in care facilities (Kim and Hah, 1998), effects on depression, emotional stability and quality of life of the elderly with mild cognitive impairment living in the community (Han and Lee, 2002; Sung and Lee, 2016), and effects on psychological energy of the elderly in nursing homes (Choi, 2016). However, the results are insufficient and not consistent, and thus it is necessary to constantly verify the effects of validation therapy for elderly patient in nursing facilities.

It was found that non-pharmacological alternative therapy to promote health of the elderly with dementia is effective (Lee and Kim, 2009), and the effects in addition to improving cognitive functions include reducing various mental and behavioral symptoms, promoting emotional stability and alleviating psychological symptoms (Jeong and Han, 2013). Horticultural therapy helps treatment and rehabilitation of mind and body through creative activities of seeing, smelling and touching plants and interactions between horticultural therapist and patients during program, prevents dementia and geriatric diseases and helps recover memory. It is also proved effective for depression of the elderly living in nursing homes or care facilities (Yoo and Jeong, 2010), aggression (Oh et al., 2011), mental functions of the elderly with cerebral apoplexy (Lee et al., 2016), and mental health of the elderly living in care facilities (Song, 2012). However, most studies use survey tools to measure the effects. Thus, this study is attempted to verify the effects of horticultural activity programs based on validation therapy as an inter- vention program to promote mental health of the elderly living in care facilities on their mental state, using Vibraimage, which is a psychophysiological measurement tool.

\section{Research Methods}

\section{Subjects and period}

The subjects were recruited through convenience sampling after sending a notice about the research to the heads of nursing homes located in $\mathrm{A}$, and selected those who understand the purpose of research and agree to participate in the study at two nursing homes willing to participate in the program. There were total 60 subjects, 23 male and 37 female, with the average age of $79.12 \pm 6.84$. Fifteen participants were randomly allocated to each of the experimental group and control group in the two nursing homes. Total 58 participants remained as the final subjects of analysis, excluding one in the experimental group and one in the control group who could not participate in both pretest and posttest by leaving the nursing homes.

The specific selection criteria of the subjects are as follows. The subjects selected are aged 70 and above, can read and write, can voluntarily participate in the program by walking or using a wheelchair, can respond to the researcher's questions and communicate, are not diagnosed with dementia, have never participated in a similar horticultural activity program based on validation therapy before, understand the purpose of research and have agreed to participate. The subjects were explained that they could discontinue anytime in the middle of the program even though they agreed to participate in the study. The research period was from March 15 to November 22, 2018, and the pretest was conducted before starting Session 1 of the program and the posttest after finishing Session 10. This study obtained approval in the deliberation process of the IRB (2014-08-008-001) in the university hospital to which the researcher belongs. 


\section{Tools}

\section{Horticultural activity program based on validation therapy}

The horticultural activity program applied to the experimental group was designed to heighten positive emotions, increase psychological energy and promote mental health by implementing validation therapy in the interactions with the subjects made in all activities of the program. The program was carried out once a week at 11 a.m., 50 minutes per session, in total 10 sessions at the group activity room of the two nursing homes.

The activities for each session of the program are divided into three stages: introduction, development and wrap-up. The introduction stage was comprised of reality orientation training that improves memory functions by asking questions about what time it is, where they are, and who the host is, as well as brain strength exercises developed by the National Health Insurance Service for long-term care nursing home patients. In the development stage, skills of validation therapy were applied to the horticultural activities of each session to produce outputs. The wrap-up stage was carried out by sharing thoughts and feelings about the activities.

Feil's validation therapy was developed to reduce anxiety and stress of elderly patients with dementia and promote their security and self-esteem. This program applied five of the techniques, such as the five principles, repeating technique, recalling technique, sensory technique, and physical contact technique (Kim et al., 2012). The five principles are to make conversations with the subjects using non-threatening and specific words about who, what, when, where and how except 'why' and to encourage participants to express their emotions through realistic questions. The subjects are never asked 'why' throughout the activities of each session, but are asked only to express or make 'what'. The repeating technique is based on how the elderly feels comfortable when hearing others repeat what they said. When the elderly subjects talk about the plant names and feelings about participating in the activities, their key words or expressions are repeated back in a similar tone of voice and speed. The recalling technique encourages the subjects to recall the memories of the past, which is a characteristic of senescence, inducing experiences of growing flowers and plants or memories about cultivation in the past, thereby enabling them to examine how the plants remain in the memories and reestablish the familiar adaptation mechanism. The sensory technique determines the sensory channels mostly used by the elderly and induces them to communicate by using the remaining sensory functions. Since horticultural activities improve emotional functions by using the five senses, the activity to improve at least one of the five senses (sight, smell, touch, taste, hearing) is planned for each session. The senses of touch and smell are stimulated by making hand sanitizer in Session 1 and making potpourri and planting rosemary in Session 2; the sense of touch by planting flowers and plants from Sessions 3 to 5; the sense of touch by making a topiary, wreath and frame of pressed flower from Sessions 6 to 8; and the sense of taste by cooking with wheat seeds in Sessions 9 and 10. Moreover, the subjects were encouraged to sing old songs with the background music along with visual functions of examining the ingredients for plant activities throughout all sessions, thereby using the sense of hearing. In particular, the horticultural therapist determined the sensory channel preferred by each subject in every session, using the sensory functions in communication. The physical contact technique was provided by making physical conduct such as holding the hand of the elderly participants at least once or placing an arm around their shoulders during the activities of each session. Communication with the subjects was conducted by making eye contact, and activities are directed in a clear pronunciation and a calm, friendly and low tone of voice. Table 1 shows the horticultural activities of each session.

Table 1. Validation horticultural activity program

\begin{tabular}{cl}
\hline Session & \multicolumn{1}{c}{ Horticultural activity } \\
\hline 1 & Making hand sanitizer \\
2 & Making potpourri and planting rosemary \\
3 & Planting fleshy plants \\
4 & Planting Epipremnum aureum plants \\
5 & Planting kalanchoe plants \\
6 & Making a topiary \\
7 & Making a wreath \\
8 & Making a frame of pressed flower \\
9 & Planting wheat seeds \\
10 & Cooking wheat vegetable pancakes \\
\hline
\end{tabular}




\section{Assessment tool}

Vibraimage (ELSYS, 2014) analyzes the psychophysiological and emotional state by taking an image of the micromovement and balancing of head based on the vestibular-emotional reflex (VER) that explains the relevance between mental function and brain reflex-control mechanism. Vibraimage measures the mediator variables of mental functions that can be controlled through vibration variation and frequency. Mediator variables are divided into 10 variables such as aggression, stress, tension, suspect, balance, charm, energy, self-regulation, inhibition, and neuroticism, and mental function state in the positive, negative and physiological domain(Choi, 2017).

This study used Vibraimage 8 PRO which is a bio-signal measurement system developed by ELSYS (2014), to measure the mental functions. This tool analyzes the emotionalpsychological state of people through visual data by filming the sitting subject for 1 minute with a digital web camera using non-contact equipment. It perceives fine vibrations of the human body, changes them to electric signals and visualizes them. The 10 psychophysiological mediator variables are scored this way, and higher scores indicate higher attributes of the relevant variables.

\section{Data analysis}

Data collected was computerized and statistically analyzed using the IBM SPSS Statistics 20.0 program, and the specific statistical method is as follows. A t-test was conducted for the preliminary test of homogeneity of the experimental and control group, and paired t-test and t-test were conducted to compare the results of the two groups before and after the experiment.

\section{Results and Discussion}

\section{Preliminary test of homogeneity}

In all of aggression, stress, tension, suspect, balance, charm, energy, self-regulation, inhibition, neuroticism and positive, negative and physiological domains of the two groups before the horticultural activity program based on validation therapy, the mean difference between the two groups was not statistically significant. Therefore, the two groups were homogeneous (Table 2).

Table 2. Homogeneity of mental function state of two groups

\begin{tabular}{|c|c|c|c|c|c|c|}
\hline \multirow{2}{*}{ Sub-factor } & \multicolumn{2}{|c|}{ Exp } & \multicolumn{2}{|c|}{ Con } & \multirow{2}{*}{$\mathrm{t}$} & \multirow{2}{*}{$p$} \\
\hline & M & SD & M & SD & & \\
\hline Aggression & 44.12 & 6.66 & 45.77 & 6.91 & .923 & .360 \\
\hline Stress & 23.63 & 6.88 & 23.05 & 8.04 & -.295 & .769 \\
\hline Tension & 19.75 & 5.85 & 19.91 & 7.83 & .087 & .931 \\
\hline Suspect & 29.31 & 3.77 & 29.90 & 3.78 & .591 & .557 \\
\hline Balance & 70.00 & 8.34 & 67.00 & 11.25 & -1.152 & .254 \\
\hline Charm & 78.19 & 5.86 & 76.96 & 7.47 & -.697 & .489 \\
\hline Energy & 34.40 & 9.94 & 39.82 & 11.41 & 1.929 & .059 \\
\hline Self- regulation & 73.41 & 6.54 & 71.64 & 6.40 & -1.039 & .303 \\
\hline Inhibition & 18.52 & 2.82 & 19.47 & 4.40 & .977 & .333 \\
\hline Neuroticism & 31.64 & 10.90 & 37.17 & 13.93 & 1.682 & .098 \\
\hline Positive & 63.98 & 5.44 & 63.89 & 5.09 & -.067 & .947 \\
\hline Negative & 29.38 & 3.75 & 29.69 & 3.74 & .316 & .753 \\
\hline Physiological & 25.08 & 6.28 & 28.32 & 8.45 & 1.658 & .103 \\
\hline
\end{tabular}

Note. Experimental group(Exp), n=29; Control group(Con), n=29. 


\section{Pretest-posttest comparison of the experimental group and control group}

Table 3 shows the change in mean scores of the two groups before and after the horticultural activity program based on validation therapy.

For aggression, the score of the experimental group decreased from $44.12 \pm 6.66$ to $43.48 \pm 8.11(p=.754)$, and that of the control group also decreased from $45.77 \pm 6.91$ to $44.75 \pm 7.97(p=.584)$, but there was no statistical significance.
This was not consistent with the result by Choi (2016) that applied the horticultural activity program based on validation therapy to elderly patients in nursing homes, measured their anger with Vibraimage, and discovered that the aggression score has decreased. It was also not consistent with Sung and Lee (2016) that applied group validation therapy to patients with mild dementia at a day care center and used the survey method that did not use the same tool for measurement but reported that aggression has decreased. The study by Choi (2016) included patients with dementia

Table 3. Changes in mental function states before and after the validation horticultural therapy program

\begin{tabular}{|c|c|c|c|c|c|c|c|c|c|}
\hline \multicolumn{2}{|c|}{ Sub-factor } & \multicolumn{2}{|c|}{ Pre } & \multicolumn{2}{|c|}{ Post } & \multirow{2}{*}{$\mathrm{t}^{\mathrm{z}}$} & \multirow{2}{*}{$p$} & \multirow{2}{*}{$\mathrm{t}^{\mathrm{y}}$} & \multirow{2}{*}{$p$} \\
\hline 5uo-1a & & M & SD & M & SD & & & & \\
\hline \multirow{2}{*}{ Aggression } & Con & 45.77 & 6.91 & 44.75 & 7.97 & .554 & .584 & \multirow{2}{*}{.601} & \multirow{2}{*}{.550} \\
\hline & Exp & 44.12 & 6.66 & 43.48 & 8.11 & .317 & .754 & & \\
\hline \multirow{2}{*}{ Stress } & Con & 23.05 & 8.04 & 23.60 & 6.75 & -.320 & .752 & \multirow{2}{*}{-1.619} & \multirow{2}{*}{.111} \\
\hline & Exp & 23.63 & 6.88 & 26.66 & 7.64 & -1.727 & .095 & & \\
\hline \multirow{2}{*}{ Tension } & Con & 19.91 & 7.83 & 33.82 & 20.44 & -3.544 & $.001 *$ & \multirow{2}{*}{-.885} & \multirow{2}{*}{.380} \\
\hline & Exp & 19.75 & 5.85 & 38.39 & 18.91 & -4.879 & $.000^{* *}$ & & \\
\hline \multirow{2}{*}{ Suspect } & Con & 29.90 & 3.78 & 34.06 & 6.43 & -2.974 & $.006^{*}$ & \multirow{2}{*}{-1.293} & \multirow{2}{*}{.201} \\
\hline & Exp & 29.31 & 3.77 & 36.19 & 6.10 & -4.925 & $.000 * *$ & & \\
\hline \multirow{2}{*}{ Balance } & Con & 67.00 & 11.25 & 63.07 & 11.76 & 1.587 & .124 & \multirow{2}{*}{-1.210} & \multirow{2}{*}{.232} \\
\hline & Exp & 70.00 & 8.34 & 66.38 & 8.89 & 1.486 & .148 & & \\
\hline \multirow{2}{*}{ Charm } & Con & 76.96 & 7.47 & 74.49 & 8.85 & 1.168 & .253 & \multirow{2}{*}{-.544} & \multirow{2}{*}{.589} \\
\hline & Exp & 78.29 & 5.86 & 75.67 & 7.62 & 1.773 & .087 & & \\
\hline \multirow{2}{*}{ Energy } & Con & 39.82 & 11.41 & 36.87 & 9.81 & 1.361 & .184 & \multirow{2}{*}{1.651} & \multirow{2}{*}{.104} \\
\hline & Exp & 34.40 & 9.93 & 32.52 & 10.22 & .706 & .486 & & \\
\hline \multirow{2}{*}{ Self-regulation } & Con & 71.64 & 6.40 & 68.53 & 6.71 & 1.706 & .099 & \multirow{2}{*}{-1.379} & 174 \\
\hline & Exp & 73.41 & 6.54 & 70.99 & 6.89 & 1.449 & .158 & & .174 \\
\hline Inhibition & Con & 19.47 & 4.40 & 17.03 & 4.71 & 2.315 & $.028^{*}$ & 007 & 368 \\
\hline 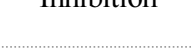 & Exp & 18.52 & 2.82 & 15.98 & 4.12 & 2.697 & $.012 *$ & .01 & .500 \\
\hline Neuroticism & Con & 37.17 & 13.93 & 30.87 & 19.75 & 1.707 & .099 & 1789 & 079 \\
\hline & Exp & 31.64 & 10.94 & 22.87 & 13.79 & 3.269 & $.003^{*}$ & & \\
\hline Positive & Con & 63.89 & 5.09 & 60.74 & 5.48 & 2.207 & $.036^{*}$ & -431 & 668 \\
\hline 1 Usiltive & Exp & 63.98 & 5.45 & 61.39 & 6.02 & 1.998 & .056 & - & .000 \\
\hline Negative & Con & 29.69 & 3.74 & 34.06 & 6.61 & -3.117 & $.004 *$ & -1.271 & .209 \\
\hline & Exp & 29.38 & 3.75 & 36.18 & 6.21 & -5.159 & $.000^{* *}$ & & \\
\hline Physiological & Con & 28.32 & 8.45 & 23.95 & 12.01 & 1.949 & .061 & 1641 & 106 \\
\hline & Exp & 25.08 & 6.27 & 19.42 & 8.80 & 3.244 & $.003^{*}$ & 1.041 & .100 \\
\hline
\end{tabular}

Note. Experimental group(Exp), $\mathrm{n}=29$; Control group(Con), $\mathrm{n}=29$.

${ }^{\mathrm{z}}$ Paired t-test between pre and post program within the group.

${ }^{\mathrm{y}}$ Paired t-test between groups after the program.

${ }^{*} p<.05,{ }^{* *} p<.001$. 
at one nursing home, but this study is different in that patients with dementia are excluded from the subjects. While homogeneity was secured in the scores of effect measurement variables and age of the subjects recruited from the two facilities, exogenous variables according to the internal/external environment of the facility and other characteristics were not controlled, which may have affected the results. Therefore, it is necessary to further elaborate the research design and replicate the experiment. Stress increased the experimental group from $23.63 \pm 6.88$ to $26.66 \pm 7.64$ $(p=.095)$ and in the control group from $23.05 \pm 8.04$ to $23.60 \pm 6.75(p=.752)$, but there was no statistical significance. Tension showed a statistically significant increase in the experimental group from $19.75 \pm 5.85$ to $38.39 \pm 18.91(p<.001)$, and also in the control group from $19.91 \pm 7.83$ to $33.82 \pm 20.44$ $(p=.001)$. This supports the report that tension increases in senescence due to physical aging and increased rigidity (Korean Neuros Pyschiatric Associaton, 2017). The horticultural activity program based on validation therapy could not alleviate tension, and in the fact that the participants feel anxious when their tension is high, this result did not support Koh (2017) that used the survey method instead of the same assessment tool but reported that the horticultural activity of raising companion plants was effective for anxiety, which is a sub-factor of mental health, for the elderly living alone. It is necessary to later verify the effects of a program adjusted so that it does not increase tension about new activities by significantly lowering the intensity of activity for the elderly patients in long-term care nursing homes while using the same horticultural activities. Suspect showed a statistically significant increase in the experimental group from $29.31 \pm 3.77$ to $36.19 \pm 6.10(p<.001)$, and also in the control group from $29.90 \pm 3.78$ to $34.06 \pm 6.43$ $(p=.006)$. This was different from the results of Sung and Lee (2016) reporting that when Schrijnemaekers et al. (2002) applied group validation therapy, it did not have a statistically significant effect on controlling problem behavior of patients with dementia, but in clinical observation there was a positive change in mental and behavioral symptoms and had motivation to control negative behavior, with which this study combined horticultural activities. This may be because both groups could not be relieved as they become more suspicious. Thus, it is necessary to conduct research on the effects when extending the short-term 10 -session program to a long-term program for elderly patients in nursing homes aged 70 and above on average to secure the time to build trust with the subjects.

Balance decreased in the experimental group from $70.00 \pm 8.34$ to $66.38 \pm 8.89(p=.148)$, and also in the control group from $67.00 \pm 11.25$ to $63.07 \pm 11.76(p=.124)$, but there was no statistical significance. This was not consistent with Hwang and Yeo (2001) that did not use the same assessment tool but used the survey-type emotional balance scale and reported that the emotional balance scores of 8 elders with dementia increased after horticultural therapy. Since the elderly patients in this study were not diagnosed with dementia, they may have shown different responses depending on their cognitive functions toward validation therapy that acknowledges everything about their current state without asking why. Accordingly, it is necessary to measure the effects later in consideration of cognitive functions. Charm decreased in the experimental group from 78.29 \pm 5.86 to $75.67 \pm 7.62(p=.087)$, and also in the control group to $76.96 \pm 74.49(p=.253)$, but there was no statistical significance. Energy decreased in the experimental group from $34.40 \pm 9.93$ to $32.52 \pm 10.22(p=.486)$, and also in the control group from $39.82 \pm 11.41$ to $36.87 \pm 9.81(p=.184)$, but there was no statistical significance. This result was not consistent with Choi (2016) that the horticultural therapy program based on validation therapy significantly increased energy of the elderly in facilities. This is in line with the result that did not support the result about aggression. This study is different in that it excluded patients with dementia, and thus it is necessary to repeatedly carry out this program considering cognitive functions in the future. Self-regulation decreased in the experimental group from $73.41 \pm 6.54$ to $70.99 \pm 6.89$ ( $p=.158$ ), and also in the control group from $71.64 \pm 6.40$ to $68.53 \pm 6.71(p=.099)$, but there was no statistical significance. This was not consistent with Kim (2017) reporting a significant difference in self-regulation from the brain function test through electrocorticography on the elderly with mild cognitive impairment by combining forest therapy with horticultural therapy and play therapy as an integrated nature-friendly program. Inhibition decreased in the experimental group from $18.52 \pm 2.82$ to $15.98 \pm 4.12(p=.012)$, and also in the control group from $19.47 \pm 17.03$ to $17.03 \pm 4.71$ 
( $p=.028)$ with a statistically significant difference. Such a significant change is identified because both groups are receiving care from a facility and thus their emotional inhibition has decreased. The score decreased more in the experimental group than the control group, but there is a limitation in that the effect of the program was not verified by checking the amount of decrease according to time because by making an assessment later. Neuroticism showed a statistically significant decrease in the experimental group from $31.64 \pm 10.94$ to $22.87 \pm 13.79(p=.003)$, but the decrease in the control group from $37.17 \pm 13.93$ to $30.87 \pm 19.75(p=.099)$ was not statistically significant. This result proved that the program is effective in the neurotic psychophysiological state, and supported the results of Song (2012), who applied the horticultural therapy program once a week for total 12 sessions to promote mental health and improve physical functions of 19 elders aged 65 and above living in long-term care nursing homes. Although there was a difference in the measurement tool, the results showed that mental health condition improved in both the experimental group and control group, but the improvement was greater in the experimental group, and the program was also found effective in the sub-categories such as somatization, interpersonal sensitivity, depression, anxiety, hostility, phobic anxiety, paranoia and psychosis. Moreover, the positive domain decreased without statistical significance in the experimental group from $63.98 \pm 5.45$ to $61.39 \pm 6.02(p=.056)$, but it decreased with statistical significance in the control group from $63.89 \pm 5.09$ to $60.74 \pm 5.48(p=.036)$. Considering that there is no statistically significant difference even though the positive function decreased in the experimental group unlike the control group, the effect of this program seems to be maintaining the positive function. This supports Pyun (2019) that did not use the same assessment tool but reported in a study on healthy elderly aged 65 and above that horticultural activities had a positive impact on psychophysiological effects, and Yoon and Sung (2017) reporting that the horticultural therapy program on the elderly with mild dementia reduced depression and resulted in positive emotional state. The negative domain increased with statistical significance in the experimental group from $29.38 \pm 3.75$ to $36.18 \pm 6.21(p<.001)$, and in the control group from $29.69 \pm 3.74$ to $34.06 \pm 34.06 \pm 6.61(p=.004)$. This proved that this program was not effective in reducing negative mental functions. The physiological domain decreased with statistical significance in the experimental group from $25.08 \pm 6.27$ to $19.42 \pm 8.80(p=.003)$, and also decreased in the control group from $28.82 \pm 8.45$ to $23.95 \pm 12.01(p=.061)$, but with no statistical significance. This suggests that the physiological domain reflects self-inhibition and neuroticism and showed a significant decrease in neurotic state, and thus this program had a positive effect on mental functions.

\section{Conclusion}

Validation therapy applies the verbal communication method that does not ask why to patients with dementia and fully accepts the condition of the subjects. The elderly living in long-term care nursing homes are facing conflicts with others living there due to self-esteem and stress, and also have habitual geriatric depression that makes them wait for someone to come visit as they live in a fixed, limited space. Therefore, efforts must be constantly made to improve mental health of the elderly in long-term care nursing homes by taking a systematic and comprehensive approach. Accordingly, this study applied validation therapy that fully accepts and gives priority to respect rather than reasonably treating the elderly subjects even though they are not elderly patients with dementia with clear cognitive impairment, and verified the effects through psychophysiological measurement by providing a program to improve mental health of elderly patients living in nursing homes with horticultural therapy that encourages them to understand the organic relationship with plants.

Sung and Lee (2016) applied validation therapy to the elderly with mild dementia living in the community and found out that it improved the quality of life. There were many studies on horticultural therapy in terms of depression or psychological aspect of the elderly in long-term care nursing homes, but there was insufficient research on psychophysiological measurement of aggression, stress, tension, suspect, self-regulation, charm, balance, energy, inhibition, neuroticism, etc. of mental health, and thus this study will be helpful for improving mental health of the elderly in long-term care nursing homes. The main con- 
clusions of this study are as follows. After carrying out the program with the experimental group and control group, the analysis on the pretest-posttest difference in mental health showed that the program decreased neuroticism, maintained positive mental health, and promoted mental health in the physiological domain, thereby proving that the experimental group that carried out the horticultural activity program based on validation therapy showed much more improvement in mental health than the control group.

Therefore, the horticultural activity program based on validation therapy in this study can be used as a program to promote mental health of the elderly and especially help the elderly in long-term care nursing homes live a happier life. With the anticipation to use this study as the basic data to improve mental health of the elderly in long-term care nursing homes, the following suggestions can be made, in addition to the need for follow-up research. First, it is necessary to carry out a long-term program by repeating the program instead of applying the short-term 10-session program to verify the effect and durability of the program. Second, a replication study is needed with the individual characteristics controlled, such as whether the subjects have dementia in cognitive skills or their linguistic ability, by selecting the age of elderly subjects to $60 \mathrm{~s}, 70 \mathrm{~s}, 80 \mathrm{~s}$ and older to improve effectiveness of the program.

\section{References}

Choi, Y.H. 2017. VibraEmotion: Visual expression of hidden emotion based on VibraImage. Master's thesis, Sogang University, Seoul, Korea.

Choi, Y.Y. 2016. Effects of validation horticultural therapy program on the aggression and mental energy for elderly in senior care center. Master's thesis, Dankook University, Cheonan, Korea.

ELSYS. 2014. Vibraimage. Retrieved from http://www.els ys.ru/vibraimage_e.php

Han, J.S. and K.S. Lee. 2002. Effects of the horticultural therapy on quality of life and depression in the institutionalized elderly. J. Korean Acad. Psychiatr. Ment. Health Nurs. 11(4):609-620.

Hwang, I.O. and C.O. Yeo. 2001. Effect of horticultural therapy on improvement in emotional functions of the elderly with dementia. J. Busan Women Coll. 23:347-374.

Jeong, H.G. and C.S. Han. 2013. Diagnosis and treatment of dementia in primary care. J. Korean Med. Assoc. 56(12):1104-1112.

Kim, C.M. 2017. Development and effect analysis of nature-friendly integrated program for the elderly with mild cognitive impairment. Doctoral dissertation, Sun Moon University, Asan, Korea.

Kim, E.J., M.J. Kwon, M.J. Kim, S.N. Kim, H.Y. Kim, Y.I. Byun, Y.K. Oh, K.J. Yoo, S.O. Lee, E.H. Lee, and M.K. Jeon. 2012. Counseling of nursing. Seoul, Korea: Jungdam Media.

Kim, K.A. and Y.S. Hah. 1998, The effect of validation therapy on behavior and emotion of elderly demented people. J. Korean Acad. Psychiatr. Ment. Health Nurs. 7(2):384-397.

Koh, E.H. 2017. Effect of ornamental plants on mental health of elderly people living alone in the viewpoint of companion plants. Doctoral dissertation, Sahmyook University, Seoul, Korea.

Korean Neuro Psychiatric Association. 2017. Textbook of neuropsychiatry. Seoul, Korea: iMiS Company.

Lee, K.M. 2014. Effects of the reminiscence therapy with group art treatment on the sentiment and quality of life for the elders who live in care facilities. Master's thesis, Woosuk University, Jeonju, Korea.

Lee, S., Y.S. Son, Y.Y. Choi, and M.S. Lee. 2016. The effects of mental function of horticultural therapy program of rehabilitation of interpersonal caring for stroke elderly in care facility. J. Korean Soc. People Plants Environ. 19(Suppl. I):181-182.

Lee, S.B. and K.W. Kim. 2009. Nonpharmacological interventions for Alzheimer's disease. J. Korean Med. Assoc. 52(11):1069-1076.

Ministry of Health and Welfare. 2019. Current status of senior care and assisted living facilities in Korea. Retrieved from http://www.mohw.go.kr

Oh, K.H., H.Y. Oh, N.S. Kim, and W.K. Jo. 2011. Effect of horticultural therapy for the improvement of aggressiveness of the aged with dementia in long term care facilities, J. Korean Soc. People Plants Environ. 14(Suppl. I):194-196. 
Pyun, S.B. 2019. Effects of horticulture activity for improving brain activity and cognitive ability of elderly. Master's thesis, Kunkook University, Seoul, Korea.

Schrijnemaekers, V., E. van Rossum, M. Candel, C. Frederiks, M. Derix, H. Sielhorst, and P. van den Brandt. 2002. Effects of emotion-oriented care on elderly people with cognitive impairment and behavioral problems. Int. J. Geriatr. Psychiatry 17(10):926-937. https://doi.org/10.10 02/gps.681

Song, H.S. 2012. A study on the effect of horticultural therapy on mental health of seniors in long-term care facilities. Master's thesis, Dongguk University, Gyeongju, Korea.

Statistics Korea. 2011. Population projections for Korea 2010-2060. Dajeon, Korea: Author.
Sung, M.R. and D.Y. Lee. 2016. Effects of community based group validation therapy on depression, quality of life, behavioral problems, and cognitive function in patients with dementia. J. Korean Gerontol. Nurs. 18(1):22-31. https://doi.org/10.17079/jkgn.2016.18.1.22

Yoo, Y.K. and H.Y. Jeong. 2010. Effect of horticultural therapy program on the depression and self-esteem of the older adult in facilities. J. Korean Soc. People Plants Environ. 13(3):7-15.

Yoon, M.J. and K.M. Sung. 2017. The effects of a horticultural program based on Cox's interaction model on ability for daily life and depression in older patients with mild dementia. Korean J. Rehabil. Nurs. 20(1):12-21. https://doi.org/10.7587/kjrehn.2017.12 